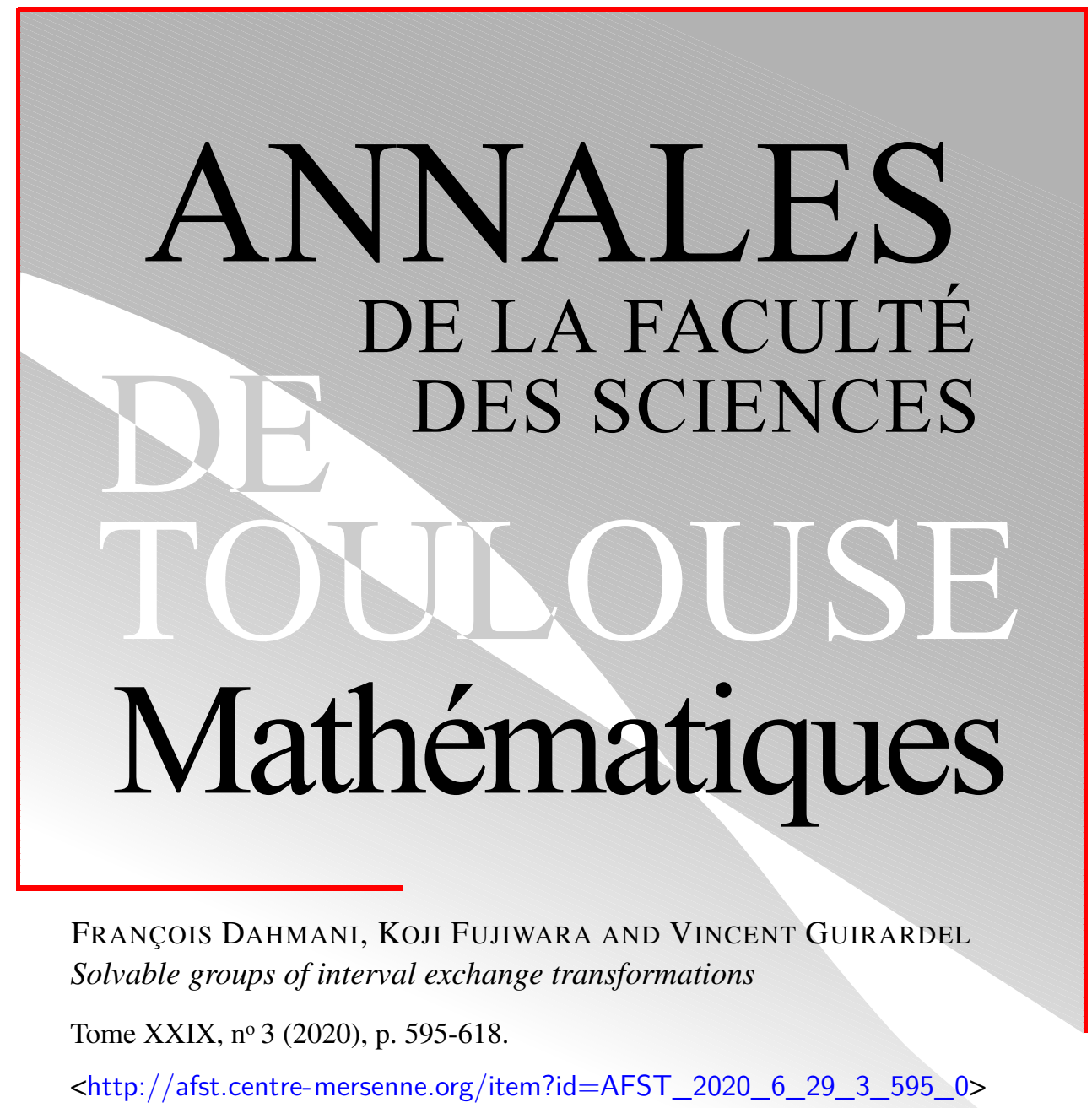

(C) Université Paul Sabatier, Toulouse, 2020, tous droits réservés.

L'accès aux articles de la revue "Annales de la faculté des sciences de Toulouse Mathématiques » (http://afst.centre-mersenne.org/), implique l'accord avec les conditions générales d'utilisation (http://afst. centre-mersenne.org/legal/). Toute reproduction en tout ou partie de cet article sous quelque forme que ce soit pour tout usage autre que l'utilisation à fin strictement personnelle du copiste est constitutive d'une infraction pénale. Toute copie ou impression de ce fichier doit contenir la présente mention de copyright.

\title{
cedram
}

Article mis en ligne dans le cadre du

Centre de diffusion des revues académiques de mathématiques

http://www.centre-mersenne.org/ 


\title{
Solvable groups of interval exchange transformations ${ }^{(*)}$
}

\author{
FRANÇOIS DAHMANI ${ }^{(1)}$, \\ Koji FuJiwara ${ }^{(2)}$ And Vincent Guirardel ${ }^{(3)}$
}

\begin{abstract}
We prove that any finitely generated torsion free solvable subgroup of the group IET of all Interval Exchange Transformations is virtually abelian. In contrast, the lamplighter groups $A \prec \mathbb{Z}^{k}$ embed in IET for every finite abelian group $A$, and we construct uncountably many non pairwise isomorphic 3 -step solvable subgroups of IET as semi-direct products of a lamplighter group with an abelian group.

We also prove that for every non-abelian finite group $F$, the group $F \imath \mathbb{Z}$ does not embed in IET.

RÉsumé. - Nous démontrons que tout sous-groupe de type fini résoluble sans torsion du groupe IET des échanges d'intervalles est virtuellement abélien. A l'opposé, les groupes d'allumeurs de réverbères $A \imath \mathbb{Z}^{k}$ se plongent dans IET pour tout groupe abélien fini $A$, et nous construisons un nombre non dénombrable de sousgroupes résolubles de classe 3 dans IET non isomorphes entre eux comme produits semi-directs d'un groupe d'allumeurs de réverbères avec des groupes abéliens.

Nous démontrons aussi que pour tout groupe fini non-abélien $F$, le produit en couronne $F \imath \mathbb{Z}$ ne se plonge pas dans IET.
\end{abstract}

\section{The group IET and its subgroups}

The group IET of interval exchange transformations is the group of all bijections of the interval $[0,1)$ that are piecewise translations with finitely many discontinuity points.

$\left({ }^{*}\right)$ Reçu le 24 janvier 2017, accepté le 16 octobre 2018.

(1) Université Grenoble Alpes, Institut Fourier, F-38000 Grenoble (France) francois.dahmani@univ-grenoble-alpes.fr

(2) Department of Mathematics, Kyoto University, Kyoto, 606-8502 (Japan) kfujiwara@math.kyoto-u.ac.jp

(3) Univ Rennes, CNRS, IRMAR - UMR 6625, F-35000 Rennes (France) vincent.guirardel@univ-rennes1.fr

Article proposé par Jean-Pierre Otal. 
Rather unexpectedly, the recent study of this group has given evidences that it is not as big as one could have thought, in several commonly accepted features. For instance, in [3] we established that IET does not have many free subgroups (if any at all), and that the connected Lie groups that can embed in it are only abelian. Another fact in this direction is that given any finitely generated subgroup of IET, and any point $x \in[0,1)$ the orbit of $x$ grows (in cardinality) at most polynomially in the word length of the elements of the subgroup [3, Lem. 6.2].

Yet another instance of these evidences is given by the main result of [7] of Juschenko and Monod which implies that certain natural subgroups of this group are amenable. More precisely, given $\alpha \in \mathbb{R} \backslash \mathbb{Q}$, the subgroup $\mathrm{IET}_{\alpha}$ of transformations whose translation lengths are all multiples of $\alpha$ modulo 1 is amenable. Indeed, given any finitely generated subgroup $G$ of $\mathrm{IET}_{\alpha}, G$ can be viewed as a group of homeomorphisms of the Cantor set $K$ obtained by blowing up the $R_{\alpha}$-orbit of the discontinuity points of the generators of $G$, where $R_{\alpha}$ is the rotation $x \mapsto x+\alpha \bmod 1$ [1]. Denoting by $\widehat{R}_{\alpha}$ the homeomorphism of $K$ induced by $R_{\alpha}$, this embeds $G$ in the full topological group of $\widehat{R}_{\alpha}$, which is amenable by [7]. This has been extended in [6] to subgroups of rational rank $\leqslant 2$, i.e. such that the subgroup of $\mathbb{Q} / \mathbb{Z}$ generated by the translation lengths of its elements does not contain $\mathbb{Z}^{3}$.

Given these evidences, we chose to investigate the possible solvable subgroups of IET.

\section{Results}

In order to describe elementary examples of subgroups of IET, let us enlarge a bit the context, and instead of interval exchange transformations on the interval $[0,1)$ we consider the group $\operatorname{IET}(\mathcal{D})$ of interval exchange transformations on a domain $\mathcal{D}$ consisting of a disjoint union of finitely many oriented circles, and oriented half-open intervals, closed on the left (see Section 1.1). This does not make change the isomorphism classes of subgroups encountered as $\operatorname{IET} \simeq \operatorname{IET}(\mathcal{D})$.

For all $n \in \mathbb{N}, \mathbb{Z}^{n}$ embeds in $\operatorname{IET}(\mathbb{R} / \mathbb{Z})$ as a group of rotations. The following general simple fact then implies that every finitely generated virtually abelian group embeds in IET.

Proposition 1 (Proposition 1.2). - Let $G$ be a group, and assume that some finite index subgroup of $G$ embeds in IET. Then so does $G$. 
It is then natural to ask which virtually polycyclic groups embed in IET. Our first result shows that only virtually abelian ones do. By a different method, Cornulier [2] also shows that a virtually polycyclic group in IET must be virtually abelian.

Theorem 2 (See Corollary 3.2). - Let $H$ be a virtually polycyclic group. Then $H$ embeds into IET if and only if it is virtually abelian.

Since a polycyclic group is virtually torsion-free, this result is in fact a corollary of the following theorem which applies to all torsion-free solvable subgroups of IET.

ThEOREM 3 (Theorem 3.1). - Every finitely generated torsion-free solvable subgroup of IET is virtually abelian.

If we allow torsion, a much greater variety of subgroups exists. The first interesting example is an embedding of the lamplighter group $L=(\mathbb{Z} / n \mathbb{Z})$ ? $\mathbb{Z}^{k}$ in IET. Note that this group $L$ is solvable (in fact metabelian), has exponential growth, and is not virtually torsion-free.

To describe this embedding, consider the domain $\mathcal{D}=(\mathbb{Z} / n \mathbb{Z}) \times(\mathbb{R} / \mathbb{Z})$, a disjoint union of $n$ circles. Choose $\Lambda \subset \mathbb{R} / \mathbb{Z}$ a subgroup isomorphic to $\mathbb{Z}^{k}$, and view $\Lambda$ as a group of synchronized rotations in $\operatorname{IET}(\mathcal{D})$, i.e. by making $\theta \in \Lambda$ act on $\mathcal{D}=(\mathbb{Z} / n \mathbb{Z}) \times(\mathbb{R} / \mathbb{Z})$ by $(i, x) \mapsto(i, x+\theta)$. Consider the interval $I=[0,1 / 2) \subset \mathbb{R} / \mathbb{Z}$, and let $\tau$ be the transformation that is the identity outside $(\mathbb{Z} / n \mathbb{Z}) \times I$, and sends $(i, x)$ to $(i+1, x)$ for $x \in I$. Then the subgroup of $\operatorname{IET}(\mathcal{D})$ generated by $\tau$ and $\Lambda$ is isomorphic to $L$. This is illustrated in Figure 4.1. More generally, taking $\mathcal{D}=A \times(\mathbb{R} / \mathbb{Z})$ for some finite abelian group $A$, this construction yields the following result:

Proposition 4 (see Propositions 4.1 and 4.2). - For any finite abelian group $A$ and any $k \geqslant 1$, the wreath product $L=A \prec \mathbb{Z}^{k}$ embeds in IET.

One could try a similar construction, replacing the abelian finite group $A$ by a non-abelian one. But the group obtained would not be the wreath product. In fact, such a wreath product cannot embed in IET as the following result shows.

TheOREM 5 (Theorem 4.4). - If $F$ is a finite group and if $F(\mathbb{Z}$ embeds as a subgroup in IET, then $F$ is abelian.

It would be interesting to know which finitely generated wreath products embed in IET. 
Starting from a subgroup $G<\operatorname{IET}(\mathcal{D})$ and a finite abelian group $A$, the construction above allows to construct subgroups of $\operatorname{IET}(\mathcal{D} \times A)$ isomorphic to $G \ltimes \mathcal{F}_{A}$ where $\mathcal{F}_{A}$ is a subgroup of the abelian group $A^{\mathcal{D}}$. We then prove that, in contrast with the torsion-free case, this construction yields a huge variety of isomorphism classes of solvable subgroups in IET.

TheOREM 6 (Theorem 4.9). - There exist uncountably many isomorphism classes of subgroups of IET that are generated by 3 elements, and that are solvable of derived length 3 .

The method we use consists of embedding many semidirect products in a way that is related to the twisted embeddings used in [6].

\section{About proofs}

The proof of Theorem 4.4 saying that $F \imath \mathbb{Z}$ does not embed into IET uses the fact that orbits in $[0,1)$ by a finitely generated subgroup of IET have polynomial growth. On the other hand, if $F \imath \mathbb{Z}=\left(\bigoplus_{n \in \mathbb{Z}} F\right) \rtimes \mathbb{Z}$ embeds in IET, $F$ and its conjugate have to commute with each other. This gives strong algebraic restrictions on the action on $F$. Using Birkhoff theorem, we show that if $F$ itself is non-commutative, then the orbit growth of $F \imath \mathbb{Z}$ has to be exponential, a contradiction.

To prove that there are uncountably many groups as in Theorem 4.9, we start with a lamplighter group $G=(\mathbb{Z} / 3 \mathbb{Z}) \gtrless \mathbb{Z}$ constructed above on $\mathcal{D}=(\mathbb{Z} / 3 \mathbb{Z}) \times(\mathbb{R} / \mathbb{Z})$, where $\mathbb{Z}$ acts on the three circles by setting a generator to act as a synchronized rotation on them with irrational angle $\alpha$. Then, we consider $\mathcal{D}^{\prime}=(\mathbb{Z} / 2 \mathbb{Z}) \times \mathcal{D}$, on which we make $G$ act diagonally. Then we choose an interval $J$ in $\{0\} \times(\mathbb{R} / \mathbb{Z}) \subset \mathcal{D}$ and define $\tau_{J}$ on $\mathcal{D}^{\prime}$ by the identity outside $(\mathbb{Z} / 2 \mathbb{Z}) \times J$, and by $(i, x) \mapsto(i+1, x)$ on $(\mathbb{Z} / 3 \mathbb{Z}) \times J \subset \mathcal{D}^{\prime}$. This is illustrated in Figure 4.2. The group $H$ generated by $G$ and $\tau_{J}$ is a homomorphic image of the wreath product $(\mathbb{Z} / 2 \mathbb{Z})<G$, but this is not an embedding. Still, the group $H$ generated by $G$ and $\tau_{J}$ has the structure of a semidirect product $G \ltimes \mathcal{F}$ where $\mathcal{F}$ is an infinite abelian group of exponent 2 . Using Birkhoff ergodic theorem, we prove that from the isomorphism class of $H$, one can read off the length of $J$ modulo a countable additive group. This proves that by varying length of $J$, we get uncountably many distinct isomorphism classes of groups $H$.

The classification of torsion-free solvable subgroups of IET in Theorem 3.1 is based on the fact that centralizers of a minimal interval exchange transformation $T$ is small. Indeed, if $T$ is an irrational rotation on a circle, then its centralizer consists of the whole group of rotations on this circle; and if 
$T$ is not conjugate to such a rotation, a theorem by Novak [10] shows that its centralizer is virtually cyclic. If $T$ is not minimal, its centralizer can be much larger: it will for instance contain a group isomorphic to IET if $T$ fixes a non-empty subinterval.

Thus, we need to understand the orbit closures of a finitely generated group, and we also need to understand how it varies when we pass to a subgroup of finite index.

A result by Imanishi [5] about the holonomy of codimension 1 foliations shows that for each finitely generated group $G<\operatorname{IET}(\mathcal{D})$, there is a partition of $\mathcal{D}$ into finitely many $G$-invariant subdomains ${ }^{(1)}$, such that in restriction to each subdomain, either every orbit is dense (such a subdomain is called an irreducible component), or every orbit is finite of the same cardinal (and one can say more, see Proposition 2.2). In particular, for each $x \in \mathcal{D}, \overline{G . x}$ is either finite, or is the closure of a subdomain (not a Cantor set).

When passing to a finite index subgroup $G_{0}$ of $G$, it could happen that an irreducible component for $G$ splits into several irreducible components for $G_{0}$.

For example, consider $\mathcal{D}=(\mathbb{Z} / 2 \mathbb{Z}) \times(\mathbb{R} / \mathbb{Z})$, and consider the subgroup $G$ of $\operatorname{IET}(\mathcal{D})$ generated by the three following tranformations $\tau, R_{0}, R_{1}$. Let $\tau$ be the involution $(i, x) \mapsto(i+1, x)$; let $\alpha \in \mathbb{R} \backslash \mathbb{Q}$ and let $R_{0}$ be the rotation of angle $\alpha$ on the circle $\{0\} \times(\mathbb{R} / \mathbb{Z})$ and as the identity on the circle $\{1\} \times(\mathbb{R} / \mathbb{Z}) ;$ and let $R_{1}=\tau R_{0} \tau^{-1}$. Then $\left\langle R_{0}, R_{1}\right\rangle \simeq \mathbb{Z}^{2}$, and $G=\left\langle\tau, R_{0}\right\rangle \simeq$ $(\mathbb{Z} \oplus \mathbb{Z}) \rtimes(\mathbb{Z} / 2 \mathbb{Z})$, and any orbit of $G$ is dense.

However, $G_{0}=\left\langle R_{0}, R_{1}\right\rangle$ is a finite index subgroup which preserves each circle. The two circles are the irreducible components of $G_{0}$. But this phenomenon cannot occur any more when passing to a further finite index $G_{1}$ of $G_{0}$ because $G_{1}$ has to contain an irrationnal rotation on each circle, thus ensuring that the two circles are still irreducible components of $G_{1}$. This group $G_{0}$ is what we call unfragmentable. The following technical result of independent interest shows that this is a general fact.

TheOREM 7 (see Theorem 2.11). - Given a finitely generated group $G<\mathrm{IET}$, there exists a finite index subgroup $G_{0}$ which is unfragmentable in the following sense:

(1) $G_{0}$ acts by the identity outside its irreducible components

(2) If $G_{1}$ is a finite index of $G_{0}$, any irreducible component of $G_{0}$ is also an irreducible component of $G_{1}$.

(1) A subdomain of $\mathcal{D}$ is a subset that consists of finitely many semi-open intervals, closed on the left 


\section{Generalities}

\subsection{Definitions}

Definition 1.1. - In all the following, a domain will be a non-empty disjoint union of finitely many oriented circles, and oriented half-open, bounded intervals (closed on the left).

Given a domain $\mathcal{D}$, the group IET $(\mathcal{D})$ of interval exchange transformations of $\mathcal{D}$ is the group of bijections of $\mathcal{D}$ that are orientation preserving piecewise isometries, left continuous with finitely many discontinuity points.

By convention, we define $\operatorname{IET}=\operatorname{IET}([0,1))$.

Given two disjoint domains $\mathcal{D}_{1}, \mathcal{D}_{2}$ having the same total length, there is an element of $\operatorname{IET}\left(\mathcal{D}_{1} \sqcup \mathcal{D}_{2}\right)$ that sends $\mathcal{D}_{1}$ on $\mathcal{D}_{2}$. We call such an element an interval exchange bijection from $\mathcal{D}_{1}$ to $\mathcal{D}_{2}$. This element then conjugates $\operatorname{IET}\left(\mathcal{D}_{2}\right)$ to $\operatorname{IET}\left(\mathcal{D}_{1}\right)$. Observe also that rescaling a domain doesn't change its group of interval exchange transformations. In particular, for any domains $\mathcal{D}_{1}, \mathcal{D}_{2}, \operatorname{IET}\left(\mathcal{D}_{1}\right)$ and $\operatorname{IET}\left(\mathcal{D}_{2}\right)$ are always isomorphic.

A subdomain $\mathcal{D}_{0} \subset \mathcal{D}$ is a subset of $\mathcal{D}$ which has finitely many connected components, and which is closed on the left. If $\mathcal{D}_{0}$ is a subdomain of $\mathcal{D}$ that is invariant by the action of $G$, then $G$ naturally maps to $\operatorname{IET}\left(\mathcal{D}_{0}\right)$ by restriction. We denote by $G_{\mid \mathcal{D}_{0}}$ its image in $\operatorname{IET}\left(\mathcal{D}_{0}\right)$. Moreover, if $\mathcal{D}=\mathcal{D}_{0} \sqcup$ $\mathcal{D}_{1}$ where both subdomains are invariant by $G$, then the induced morphism from $G$ to $\operatorname{IET}\left(\mathcal{D}_{0}\right) \times \operatorname{IET}\left(\mathcal{D}_{1}\right)$ is injective.

\subsection{Finite extensions}

Proposition 1.2. - Let $G$ be a group, and assume that some finite index subgroup of $G$ embeds in IET. Then so does $G$.

Proof. - Without loss of generality, consider $H<G$ a normal subgroup of finite index that embeds in $\operatorname{IET}(\mathcal{D})$ for some domain $\mathcal{D}$. Let $Q$ be the finite quotient $Q=G / H$. It is a classical algebraic fact (see [9]) that $G$ embeds in the wreath product $H \imath Q=H^{Q} \rtimes Q$ (where $Q$ acts on $H^{Q}$ by permuting coordinates). Thus, it suffices to show that $H^{Q} \rtimes Q$ embeds in IET.

Consider the domain $\mathcal{D}^{\prime}=Q \times \mathcal{D}$, and embed $H^{Q}$ in $\operatorname{IET}\left(\mathcal{D}^{\prime}\right)$ by making $\left(h_{q}\right)_{q \in Q}$ act on $\mathcal{D}^{\prime}=Q \times \mathcal{D}$ by $(q, x) \mapsto\left(q, h_{q} . x\right)$. Then $Q$ acts on $\mathcal{D}^{\prime}$ by left multiplication on the left coordinate. This naturally extends to a morphism $H^{Q} \rtimes Q \rightarrow \operatorname{IET}\left(\mathcal{D}^{\prime}\right)$ which is clearly one-to-one. 


\section{Irreducibility and unfragmentability for finitely generated subgroups of IET}

\subsection{IET and irreducibility}

Let $\mathcal{D}$ be a domain. Let $G=\langle S\rangle$ be a finitely generated subgroup of the group $\operatorname{IET}(\mathcal{D})$ of interval exchange transformations on $\mathcal{D}$, with $S$ symmetric.

Definition 2.1 (Irreducibility). - We say that $G$ is irreducible (on $\mathcal{D}$ ) if no subdomain of $\mathcal{D}$ is invariant under $G$.

We say that a subdomain $J$ of $\mathcal{D}$ is an irreducible component for $G$ if it is $G$-invariant, and if $G$ restricted to $J$ is irreducible.

We will see that a finitely generated group $G$ is irreducible if and only if every $G$-orbit is dense in $\mathcal{D}$ (see Corollary 2.6).

If $s \in \operatorname{IET}(\mathcal{D})$, we denote by $\operatorname{Disc}(s) \subset \dot{\mathcal{D}}$ the set of discontinuity points of $s$. If $S$ is a set of elements of $\operatorname{IET}(\mathcal{D})$, we denote by $\operatorname{Disc}(S)=\bigcup_{s \in S} \operatorname{Disc}(s)$ the set of discontinuity points of elements of $S$.

We say that $x, y \in \stackrel{\circ}{\mathcal{D}}$ are in the same regular orbit if there exist $g \in G$ continuous at $x$ with $g(x)=y$. We say that $x, y \in \stackrel{\circ}{\mathcal{D}}$ are in the same $S$ regular orbit if there exists $g_{1}, \ldots, g_{n} \in S$ such that $y=g_{n} \ldots g_{1}(x)$, and for all $i \leqslant n, g_{i-1} \ldots g_{1}(x) \in \stackrel{\circ}{\mathcal{D}} \backslash \operatorname{Disc}\left(g_{i}\right)$. We denote by $\operatorname{Reg}(x, G) \subset \stackrel{\circ}{\mathcal{D}}$ the regular orbit of $x$, and by $\operatorname{Reg}(x, S) \subset \operatorname{Reg}(x, G)$ its $S$-regular orbit. Although arguably less natural, the notion of $S$-regular orbit is the one that is needed to apply Imanishi Theorem below. On the other hand, Lemma 2.12 below will show that one can choose $S$ so that $\operatorname{Reg}(x, S)=\operatorname{Reg}(x, G)$ for all $x \in \stackrel{\circ}{\mathcal{D}}$.

Let

$$
\operatorname{Sing}(G)=\{x \in \stackrel{\circ}{D} \mid \exists g \in G, x \in \operatorname{Disc}(g)\} .
$$

By definition, $\operatorname{Sing}(G)$ does not depend on any generating set, but one easily checks that for $x \in \stackrel{\circ}{\mathcal{D}}, x \in \operatorname{Sing}(G)$ if and only if its $G$-orbit contains a point in $\operatorname{Disc}(S)$, if and only if $\operatorname{Reg}(x, S)$ contains a point in $\operatorname{Disc}(S)$. Thus, $\operatorname{Sing}(G)$ is a union of at most \# $\operatorname{Disc}(S) S$-regular orbits. We also note that for all $x \in \stackrel{\circ}{\mathcal{D}} \backslash \operatorname{Sing}(G), \operatorname{Reg}(x, S)=\operatorname{Reg}(x, G)=G . x \cap \stackrel{\circ}{\mathcal{D}}$.

We denote by $E(G) \subset \operatorname{Sing}(G)$ the set of points $x \in \operatorname{Sing}(G)$ whose regular orbit is finite, and by $E(S)$ the set of points $x \in \operatorname{Sing}(G)$ whose 
$S$-regular orbit is finite. We note that $E(G) \subset E(S)$ and that these sets are finite since

$$
E(S)=\bigcup_{x \in \operatorname{Disc}(S), \# \operatorname{Reg}(x, S)<\infty} \operatorname{Reg}(x, S)
$$

is a finite union of finite sets.

We will need to apply a decomposition theorem that applies for finite systems of isometries, so let us introduce the corresponding terminology (see [4]). Let $\overline{\mathcal{D}}$ be the obvious compactification of $\mathcal{D}$ as a union of compact intervals and circles. A partial isometry on $\overline{\mathcal{D}}$ is an isometry $\varphi: I \rightarrow J$ between two closed subintervals $I, J \subset \mathcal{D}$. Given a finite set $S$ of interval exchanges on $\mathcal{D}$, one can construct a system of isometries on $\overline{\mathcal{D}}$ as follows: for each $s \in S$, let $I_{1}, \ldots, I_{n_{s}}$ be the maximal connected subdomains on which $s$ is continuous. These intervals define a partition of $\mathcal{D}$. For each $i \leqslant n_{s}$, we let $\varphi_{i}$ be the partial isometry defined on $\bar{I}_{i}$ that extends $s_{\mid I_{i}}$. Thus, for each element of $s$, we have a finite collection of partial isometries, and we denote by $X$ the collection of all partial isometries of $\overline{\mathcal{D}}$ obtained from all the elements of $S$ in this way. Orbits of $X$ are defined in the natural way. Clearly, each $G$-orbit is contained in an $X$-orbit. One defines $\stackrel{\circ}{X}$-orbits similarly using the restriction of all the partial isometries to the interior of their domains. Then for all $x \in \stackrel{\circ}{D}$, its $\stackrel{\circ}{X}$-orbit coincides exactly with its $S$-regular orbit $\operatorname{Reg}(x, S)$.

Specifying $[4$, Thm. 3.1$]$ to our setting, we get:

Proposition 2.2 (Imanishi theorem [5], see [4, Thm. 3.1]). — Let $S$ be a finite symmetric set of interval exchanges on $\mathcal{D}$, and $G=\langle S\rangle<\operatorname{IET}(\mathcal{D})$.

Then $\stackrel{\circ}{\mathcal{D}} \backslash E(S)=\stackrel{\circ}{\mathcal{D}}_{1} \sqcup \cdots \sqcup \stackrel{\circ}{\mathcal{D}}_{p}$ where each $\stackrel{\circ}{\mathcal{D}}_{i}$ is an open subset of $\mathcal{D} \backslash E(S)$ invariant under $S$-regular orbits and such that for each $i \leqslant p$, one of the following holds:

(1) every $S$-regular orbit in $\stackrel{\circ}{\mathcal{D}}_{i}$ is dense in $\stackrel{\circ}{\mathcal{D}}_{i}$

(2) every $S$-regular orbit in $\stackrel{\circ}{\mathcal{D}}_{i}$ is finite, of the same cardinality, and $\stackrel{\circ}{\mathcal{D}}_{i} \cap \operatorname{Sing}(G)=\emptyset$.

Remark 2.3. - Theorem 3.1 of [4] is stated for systems of isometries on a finite union of intervals, but generalizes immediately to the case where we allow circles in the domain (alternatively, we could restrict to this case by cutting $\mathcal{D}$ along a point in each circle of $\mathcal{D}$ ). Our assertion 2 thus includes the possibility that $\stackrel{\circ}{\mathcal{D}}_{i}$ is a disjoint union of circles, and that all elements of $G$ act continuously on $\mathcal{D}_{i}$. Since all elements of IET preserve the orientation, there cannot be any twisted family of finite orbits in the sense of [4, Thm. 3.1]. 
Corollary 2.4. - Let $G<\operatorname{IET}(\mathcal{D})$ be a finitely generated group.

Then $\mathcal{D}$ decomposes into $G$-invariant subdomains $\mathcal{D}=\mathcal{D}_{\infty} \sqcup \mathcal{D}_{\text {fin }}$ with $\mathcal{D}_{\infty}=I_{1} \sqcup \cdots \sqcup I_{r}$ and $\mathcal{D}_{\text {fin }}=J_{1} \sqcup \cdots \sqcup J_{t}$ where each $I_{i}$ and $J_{j}$ is a $G$ invariant subdomain whose boundary is contained in $E(S) \cup \partial \mathcal{D}$, and such that

(1) for all $i \leqslant r$, every $G$-orbit in $I_{i}$ is dense in $I_{i}$

(2) for all $j \leqslant r, G$ acts on $J_{j}$ with finite orbits, all of the same cardinality; the restriction to $J_{j}$ of any $g \in G$ is continuous, and in particular, $G$ permutes the connected components of $J_{j}$.

Moreover, the collection of irreducible components of $G$, $\operatorname{Irred}(G)=$ $\left\{I_{1}, \ldots, I_{r}\right\}$, is unique.

Remark 2.5. - Note that whereas $\operatorname{Irred}(G)=\left\{I_{1}, \ldots, I_{r}\right\}$ is uniquely defined, we don't claim that the decomposition of the complement $\mathcal{D} \backslash\left(\bigcup_{i} I_{i}\right)$ into $G$-invariant subdomains upon which $G$ acts as a finite group is unique (although one could easily construct such a canonical decomposition).

Proof. - Take $S$ a finite symmetric generating set of $G$. Write $\stackrel{\circ}{\mathcal{D}} \backslash E(S)=$ $\stackrel{\circ}{\mathcal{D}}_{1} \sqcup \cdots \sqcup \stackrel{\circ}{\mathcal{D}}_{p}$ as in Proposition 2.2. Let $\mathcal{D}_{i} \subset \mathcal{D}$ be the smallest subdomain of $\mathcal{D}$ containing $\stackrel{\circ}{\mathcal{D}}_{i}$ (i.e. the set of points to which are limits of points in $\stackrel{\circ}{\mathcal{D}}_{i}$ from the right), so that $\mathcal{D}=\mathcal{D}_{1} \sqcup \cdots \sqcup \mathcal{D}_{p}$. Then $\mathcal{D}_{i}$ is $G$-invariant because if $g \mathcal{D}_{i} \cap \mathcal{D}_{j} \neq \emptyset$, then $\mathcal{D}_{i} \cap g^{-1} \mathcal{D}_{j}$ contains an interval and for any point $x \in \stackrel{\circ}{\mathcal{D}} \backslash \operatorname{Sing}(G)$ in this interval, we have that $g(x) \in \operatorname{Reg}(x, S) \cap$ $\mathcal{D}_{j}$ contradicting that $\stackrel{\circ}{\mathcal{D}}_{i}$ is invariant under $S$-regular orbits. We denote by $I_{1}, \ldots, I_{r}, J_{1}, \ldots, J_{t}$ the subdomains of $\mathcal{D}_{1}, \ldots, \mathcal{D}_{p}$ (with $r+t=p$ ) according to whether they satisfy the first or the second assertion of Proposition 2.2

Then for all $i \leqslant r$ and every $x \in \stackrel{\circ}{I}_{i}$, its $G$-orbit is dense in $I_{i}$ because it contains $\operatorname{Reg}(x, S)$. If $x \in I_{i} \backslash \stackrel{\circ}{I}_{i}$, then its $G$-orbit has to contain a point in $\stackrel{\circ}{I}_{i}$. Indeed, otherwise, the $G$-orbit of $x$ would be finite, and so would be the $G$-orbits of points $x^{\prime}$ in a right neighbourhood of $x$, contradicting that regular orbits are dense in $\stackrel{\circ}{I}_{i}$.

For $j \leqslant t$, then since $\stackrel{\circ}{J}_{j} \cap \operatorname{Sing}(G)=\emptyset, G$ acts continously on $\stackrel{\circ}{J}_{j}$, so $g_{\mid J_{j}}$ is continuous for every $g \in G$.

The uniqueness of $\left\{I_{1}, \ldots, I_{r}\right\}$ is immediate from the fact that $I_{i}$ is $G$ invariant and that every $G$-orbit is dense in $I_{i}$.

The following corollary is clear. 
Corollary 2.6. - A subgroup $G<\operatorname{IET}(\mathcal{D})$ is irreducible if and only if every $G$-orbit is dense in $\mathcal{D}$.

Remark 2.7. - In the case where $E(S)=\emptyset$, each subdomain $I_{i}, J_{j}$ appearing in Corollary 2.4 is a union of connected components of $\mathcal{D}$. We can always cut the domain $\mathcal{D}$ to reduce to this situation as follows.

We say that we cut a domain $\mathcal{D}_{1}$ along a finite set of points if we map it to some domain $\mathcal{D}_{2}$ by an interval exchange bijection $\tau: \mathcal{D}_{1} \rightarrow \mathcal{D}_{2}$ that is discontinuous exactly on this set of points, and such that $\tau^{-1}$ is continuous. For instance, if $\mathcal{D}_{1}=[a, b)$, cutting along $c \in[a, b)$ yields a domain $\mathcal{D}_{2}=$ $[a, c) \sqcup[c, b)$, and if $\mathcal{D}_{1}$ is a circle, cutting along one point yields a domain that is a half-open interval. If $G_{1} \subset \operatorname{IET}\left(\mathcal{D}_{1}\right)$, we then get by conjugation a group $G_{2} \subset \operatorname{IET}\left(\mathcal{D}_{2}\right)$ (in particular, $G_{2}$ is isomorphic to $G_{1}$ ).

Now consider $G<\operatorname{IET}(\mathcal{D})$, and $S$ is a symmetric generating set of $G$. Then cutting $\mathcal{D}$ along the (finitely many) points in $E(S)$ yields a domain $\mathcal{D}^{\prime}$ and a group $G^{\prime}<\operatorname{IET}\left(\mathcal{D}^{\prime}\right)$ conjugate to $G$ by an interval exchange bijection from $\mathcal{D}$ to $\mathcal{D}^{\prime}$ such that $E\left(S^{\prime}\right)=\emptyset$ (where $S^{\prime}$ is the conjugate of $S$ ).

\subsection{Virtual unfragmentability for finitely generated groups of IET}

Let $G$ be a finitely generated subgroup of $\operatorname{IET}(\mathcal{D})$.

Definition 2.8 (Unfragmentability). - We say that $G$ is unfragmentable if for any subdomain $J \subset \mathcal{D}$ which is invariant by a finite index subgroup of $G, J$ is $G$-invariant.

We say that an element $a \in \operatorname{IET}(\mathcal{D})$ is unfragmentable if the cyclic group $\langle a\rangle$ is unfragmentable.

Here is an equivalent definition.

LEMMA 2.9. - The subgroup $G$ is unfragmentable if each of its finite orbits is trivial, and if, for every irreducible component $J$ of $G$, and every finite index subgroup $H$ of $G$, the restriction of $H$ on $J$ is irreducible.

Proof. - Let $I_{1}, \ldots, I_{r} \subset \mathcal{D}$ be the irreducible components of $G$, and $I^{\prime}=\mathcal{D} \backslash\left(I_{1} \cup \cdots \cup I_{r}\right)$.

Assume that $G$ is unfragmentable. If $G$ has a non-trivial finite orbit (necessarily in $I^{\prime}$ ), then there exists a subdomain $J \subset I^{\prime}$ and $g \in G$ such that $g J \neq J$. Then $J$ is a subdomain that is invariant under the finite index subgroup of $G$ acting trivially on $I^{\prime}$, but not $G$-invariant, a contradiction. If some finite index subgroup $G_{0}<G$ does not act irreducibly on some $I_{i}$, then 
there is a $G_{0}$-invariant subdomain $J \varsubsetneqq I_{i}$ and $J$ is not $G$-invariant because $I_{i}$ is an irreducible component of $G$, a contradiction.

Conversely, assume that the statement in the lemma holds and let $J \subset \mathcal{D}$ be a subdomain invariant under a finite index subgroup $G_{0}<G$. Since $G$ acts trivially on $I^{\prime}, J \cap I^{\prime}$ is $G$-invariant. For each $i, J \cap I_{i}$ is $G_{0}$-invariant, and since by assumption $G_{0}$ acts irreducibly on $I_{i}$, we either get that $J \cap I_{i}$ is empty or $J=I_{i}$, in particular, $J \cap I_{i}$ is $G$-invariant. Since this holds for each $i, J$ is $G$-invariant.

Remark 2.10. - If $\mathcal{D}$ and $\mathcal{D}^{\prime}$ are two domains with an interval exchange bijection $\tau$ from $\mathcal{D}$ to $\mathcal{D}^{\prime}$, then a group $G<\operatorname{IET}(\mathcal{D})$ is unfragmentable (resp. irreducible) if and only if its conjugate by $\tau$ in $\operatorname{IET}\left(\mathcal{D}^{\prime}\right)$ is unfragmentable (resp. irreducible).

The main theorem of this section is the following. We will use it only in the case of a cyclic group, but the general statement seems to be of interest.

THEOREM 2.11. - If $G<\operatorname{IET}(\mathcal{D})$ is a finitely generated subgroup of $\operatorname{IET}(\mathcal{D})$, it admits a finite index subgroup that is unfragmentable.

Before proving the theorem, we prove that one can find a finite symmetric generating set $S$ of $G$ such that for every $x \in \stackrel{\circ}{\mathcal{D}}, \operatorname{Reg}(x, S)=\operatorname{Reg}(x, G)$, and $E(S)=E(G)$.

Lemma 2.12. - Let $G$ be a finitely generated subgroup of $\operatorname{IET}(\mathcal{D})$.

Then there exists a finite symmetric generating set $S$ such that for all $x \in \stackrel{\circ}{\mathcal{D}}, \operatorname{Reg}(x, S)=\operatorname{Reg}(x, G)$.

Proof. - If $x \in \stackrel{\circ}{\mathcal{D}} \backslash \operatorname{Sing}(G)$, then for all generating $S$ of $G, \operatorname{Reg}(x, S)=$ $G x=\operatorname{Reg}(x, G)$. Let $S$ be a finite symmetric generating set of $G$. We are going to increase $S$ so that the Lemma holds. Fix a point $x \in \operatorname{Sing}(G)$. Let $R_{1}, \ldots, R_{n}$ be the partition of $\operatorname{Reg}(x, G)$ into $S$-regular orbits, and choose $x_{i} \in R_{i}$ for each $i \leqslant n$. By definition of $\operatorname{Reg}(x, G)$, there exists $g_{i} \in G$ such that $g_{i} . x_{1}=x_{i}$ and $g_{i}$ is continuous at $x_{1}$. Adding $\left\{g_{2}^{ \pm 1}, \ldots, g_{n}^{ \pm 1}\right\}$ to $S$ yields a symmetric generating set $S^{\prime}$ such that $\operatorname{Reg}\left(x, S^{\prime}\right)=\operatorname{Reg}(x, G)$. Since $\operatorname{Sing}(G)$ is a union of finitely many $G$-regular orbits, one can repeat this operation finitely many times and get a generating set satisfying the lemma.

Recall that $E(S)$ (resp. $E(G)$ ) is the set of points $x \in \operatorname{Sing}(G)$ whose $S$-regular orbit $\operatorname{Reg}(x, S)$ (resp. whose $G$-regular orbit $\operatorname{Reg}(x, G)$ ) is finite. The previous Lemma gives the following.

Corollary 2.13. - There exists a finite generating set $S$ of $G$ such that $E(G)=E(S)$. 
Proof of Theorem 2.11. - After cutting $\mathcal{D}$ as in Remark 2.7, one can assume that $E_{S}(G)=\emptyset$. Apply Imanishi theorem, and write $\mathcal{D}=\mathcal{D}_{\text {fin }} \cup \mathcal{D}_{\infty}$, with $\mathcal{D}_{\infty}=I_{1} \cup \cdots \cup I_{r}$ as in Corollary 2.4.

Let $G_{1}$ be a finite index subgroup of $G$ such that $\left(G_{1}\right)_{\mid \mathcal{D}_{\text {fin }}}$ is trivial, and therefore the map $G \rightarrow\left(G_{1}\right)_{\mid \mathcal{D}_{\infty}}$ is an isomorphism. Without loss of generality, and to keep readable notations, we assume that $\mathcal{D}=\mathcal{D}_{\infty}$, i.e. that every $G_{1}$-orbit is infinite.

Let $G_{0}$ be an arbitrary finite index subgroup of $G_{1}$. The fact that $E_{S}(G)=\emptyset$ means that for every $x \in \stackrel{\circ}{\mathcal{D}}_{\infty}, \operatorname{Reg}(x, G)$ is infinite. We claim that $\operatorname{Reg}\left(x, G_{0}\right)$ is also infinite. Indeed, let $g_{i}$ be a sequence of elements of $G$ that are continuous at $x$ and such that the points $g_{i} . x$ are all distinct. Since $\left[G: G_{0}\right]<\infty$, up to extracting a subsequence, we may assume that there exists an element $a \in G$ such that $a g_{i} \in G_{0}$ for all $i$. Since $\operatorname{Disc}(a)$ is finite, $a$ is continuous at $g_{i} . x$ for $i$ large enough, so $a g_{i} . x \in \operatorname{Reg}\left(x, G_{0}\right)$. This proves our claim and shows that $E\left(G_{0}\right)=\emptyset$.

By Corollary 2.13, there exists a finite generating set $S_{0}$ of $G_{0}$ such that $E\left(S_{0}\right)=\emptyset$. Then Corollary 2.4 yields a decomposition of $\mathcal{D}$ into finitely many $G_{0}$-invariant subdomains $I_{1}, \ldots, I_{r}$ on which the action of $G_{0}$ is irreducible, and since $E\left(S_{0}\right)=\emptyset$, each $I_{i}$ is a union of connected components of $\mathcal{D}$. The number $r$ depends on $G_{0}$ but is bounded by the number of connected components of $\mathcal{D}$.

Among all possible choices of finite index subgroups $G_{0}<G_{1}$, we choose $G_{0}$ so that $r$ is maximal. Then for any $G_{0}^{\prime}<G_{0}$ of finite index, the decomposition of $\mathcal{D}$ into $G_{0}^{\prime}$-irreducible components is its decomposition into $G_{0}$-irreducible components. This shows that $G_{0}$ is unfragmentable.

\section{Commutation and solvable subgroups}

Let $\mathcal{D}$ be a domain. The main result of this section is the following.

THEOREM 3.1. - Let $G<\operatorname{IET}(\mathcal{D})$ be a finitely generated torsion free solvable group. Then $G$ is virtually abelian.

Since virtually polycyclic groups are virtually torsion-free, we get

COROLlary 3.2. - Any virtually polycyclic subgroup of IET is virtually abelian.

The theorem will be proved in several steps. We start with the following property of unfragmentable elements. 
Lemma 3.3. - Let $a \in \operatorname{IET}(\mathcal{D})$ be unfragmentable with irreducible components $\operatorname{Irred}(a)=\left\{I_{1}, \ldots, I_{r}\right\}$. If $g \in \operatorname{IET}(\mathcal{D})$ is such that gag $^{-1}$ commutes with $a$, then for all $i$, either $g\left(I_{i}\right)$ is disjoint from $I_{1}, \ldots, I_{r}$, or $g\left(I_{i}\right)$ is equal to some $I_{j}$.

Proof. - Observe that $\operatorname{Irred}\left(g_{a} g^{-1}\right)=\left\{g\left(I_{1}\right), \ldots, g\left(I_{r}\right)\right\}$. For readability we will write $a^{g}=g a g^{-1}$. Since $a$ commutes with $a^{g}$, it permutes the collection $\left\{g\left(I_{1}\right), \ldots, g\left(I_{r}\right)\right\}$, and therefore some power $a^{r !}$ preserves each $g\left(I_{j}\right)$. By unfragmentability, any subdomain preserved by $a^{r !}$ is preserved by $a$, and therefore $a$ itself preserves each $g\left(I_{j}\right)$. Similarily, $a^{g}$ preserves each $I_{j}$.

The intersection $g\left(I_{j}\right) \cap I_{i}$ is $a^{g}$-invariant, and also $a$ invariant. If $g\left(I_{j}\right) \cap$ $I_{i} \neq \emptyset$, then $g\left(I_{j}\right) \cap I_{i}=I_{i}$ by irreducibility of $a$ on $I_{i}$. Similarly, $g\left(I_{j}\right) \cap I_{i}=$ $g\left(I_{j}\right)$ by irreducibility of $a^{g}$ on $g\left(I_{j}\right)$. It follows that if $g\left(I_{j}\right) \cap I_{i} \neq \emptyset$ then $g\left(I_{j}\right)=I_{i}$. The lemma follows.

Corollary 3.4. - Let $a \in G<\operatorname{IET}(\mathcal{D})$ be a unfragmentable element with irreducible components $I_{1}, \ldots, I_{n}$. Assume that for all $g \in G, g g^{-1}$ commutes with a.

Then for each $i \leqslant n,\left\{g\left(I_{i}\right), g \in G\right\}$ is a finite collection of disjoint subdomains.

Proof. - Let $g, h \in G$. Assume that $g\left(I_{i}\right) \cap h\left(I_{i}\right) \neq \emptyset$. Then, $h^{-1} g\left(I_{i}\right) \cap$ $I_{i} \neq \emptyset$, and by Lemma 3.3, this implies that $h^{-1} g\left(I_{i}\right)=I_{i}$, hence $g\left(I_{i}\right)=$ $h\left(I_{i}\right)$. Thus $\left\{g\left(I_{i}\right), g \in G\right\}$ is a collection of disjoint subdomains. It is finite because the measure of $\mathcal{D}$ is finite.

Proposition 3.5. - Let $G$ be a subgroup of $\operatorname{IET}(\mathcal{D})$. If there exists a normal abelian subgroup of $G$, containing some irreducible and unfragmentable element, then $G$ is either abelian or virtually cyclic. In particular, $G$ is virtually abelian.

Before starting the proof, let us recall the following, from [3]. Let $g \in$ $\operatorname{IET}(\mathcal{D})$ and $d(g)$ be the number of discontinuity points of $g$ on $\mathcal{D}$. Let $\|g\|=$ $\lim \frac{1}{n} d\left(g^{n}\right)$. In [3, Cor. 2.5], we proved that $\|g\|=0$ if and only if $g$ is conjugate to a continuous transformation of some domain $\mathcal{D}^{\prime}$ consisting only of circles, and one of its powers is a rotation on each circle.

Proof. - Let $a$ be such an element in $A \triangleleft G$ (with $A$ abelian). If $\|a\|=0$, then as we mentionned in the preceeding discussion, $a$ is conjugate to a continuous transformation on some $\mathcal{D}^{\prime}$, and because it is irreducible and unfragmentable, $\mathcal{D}^{\prime}$ has to be a circle and $a$ is an irrational rotation. Then, by [3, Lem. 1.1], its centralizer is conjugate to the rotation group on $\mathcal{D}^{\prime}$, and since $G$ normalises $A$, [3, Lem. 1.1] says that $G$ must also be conjugate to a group of rotations on $\mathcal{D}^{\prime}$, hence abelian. 
Assume now that $\|a\|>0$. Since $a$ is irreducible, [10, Prop. 1.5] implies that its centraliser is virtually cyclic, hence so is $A$. Therefore $A$ has a finite automorphism group. The group $G$ acts by conjugation on $A$, and since the automorphism group of $A$ is finite, the kernel of this action has finite index in $G$. This kernel is contained in the centralizer of $a$ though, so $G$ is virtually cyclic.

Proposition 3.6. - If $G<\operatorname{IET}(\mathcal{D})$ is irreducible, and contains a normal abelian subgroup $A \triangleleft G$, with an element $a \in A$ of infinite order, then $G$ is virtually abelian.

In particular, if $G$ is finitely generated, then so is $A$.

Proof. - Up to replacing $a$ by a power, Theorem 2.11 allows us to assume that $a$ is unfragmentable. Let $I_{1}, \ldots I_{k}$ be its irreducible components.

By Corollary 3.4, $\left\{g\left(I_{1}\right), g \in G\right\}$ is a finite collection of disjoint subdomains. By irreducibility of $G$, it is a partition of $\mathcal{D}$, and we thus write $\mathcal{D}=M_{1} \sqcup \cdots \sqcup M_{l}$ with $M_{1}=I_{1}$, and $M_{i}=g_{i} I_{1}$. There is a finite index subgroup $G_{0}$ of $G$ that preserves each $M_{i}$. Let $k$ be such that $a^{k} \in G_{0}$. Then, $a^{k}$ is irreducible and unfragmentable on $I_{1}$ so Proposition 3.5 ensures that the image $G_{0 \mid I_{1}} \subset \operatorname{IET}\left(I_{1}\right)$ of $G_{0}$ under the restriction map is virtually abelian. Similarly, $\left(a^{k}\right)^{g_{i}}$ is irreducible on $M_{i}$, so $G_{0}$ has virtually abelian image in restriction to each $M_{i}$.

Since $\mathcal{D}=M_{1} \sqcup \cdots \sqcup M_{l}$, the product of restriction maps yields an injection of $G_{0}$ into $\prod_{j} \operatorname{IET}\left(M_{j}\right)$. Thus, the image of $G_{0}$ is contained in a finite product of virtually abelian groups, so $G_{0}$ is virtually abelian and so is $G$.

We finish with this lemma before proving Theorem 3.1.

Lemma 3.7. - Let $G<\operatorname{IET}(\mathcal{D})$ be a finitely generated group, and assume that it contains a torsion-free abelian normal subgroup $A$. Then $A$ is finitely generated, and there is a finite index subgroup $H$ in $G$, such that $A \cap[H, H]=\{1\}$.

Proof. - Let $\operatorname{Irred}(G)=\left\{I_{1}, \ldots, I_{k}, I_{k+1} \ldots, I_{r}\right\}$ where one has ordered the components so that the image $A_{\mid I_{i}}$ of $A$ in $\operatorname{IET}\left(I_{i}\right)$ under the restriction map is a torsion group for all $i>k$ and contains an infinite order element for $i \leqslant k$. Let $\mathcal{D}_{\text {fin }}=\mathcal{D} \backslash\left(\bigcup_{i=1}^{r} I_{i}\right)$.

For every $i \leqslant k$, by Proposition 3.6 we get that the image $G_{\mid I_{i}}$ of $G$ in $\operatorname{IET}\left(I_{i}\right)$ under the restriction map is a virtually abelian group.

In other components, the image of $A$ is a torsion group. Let us denote by $G_{1}$ the image of $G$ in $\operatorname{IET}\left(\bigcup_{i=1}^{k} I_{i}\right)$ and by $G_{2}$ the image of $G$ in $\operatorname{IET}\left(\left(\bigcup_{j=k+1}^{r} I_{j}\right) \cup \mathcal{D}_{\text {fin }}\right)$ under the restriction maps. 
This gives an embedding $\iota: G \hookrightarrow G_{1} \times G_{2}$, where $G_{1}$ is virtually abelian, and $p_{2} \circ \iota(A)$ is torsion. In particular, because $A$ is torsion-free, $p_{1} \circ \iota$ is injective in restriction to $A$.

It already follows that $A$ is finitely generated, since it embeds as a subgroup of a finitely generated virtually abelian group.

Consider $H_{1}$ an abelian finite index subgroup in $G_{1}$, and $H$ the preimage of $H_{1}$ in $G$, which is a finite index subgroup.

We saw that the map $p_{1} \circ \iota$ is injective on $A$, but it vanishes on $[H, H]$ because $p_{1} \circ \iota(H)=H_{1}$ is abelian. Therefore $A \cap[H, H]=\{1\}$, thus establishing the lemma.

Proof of Theorem 3.1. - Consider $G<\operatorname{IET}(\mathcal{D})$ a finitely generated torsion free solvable group. Then its derived series has a largest index $n$ for which $G^{(n)} \neq\{1\}$ (where $G^{(n)}=\left[G^{(n-1)}, G^{(n-1)}\right]$ and $\left.G^{(0)}=G\right)$. If $n=1$, $G$ is abelian, we thus proceed by induction on $n$.

Since $G$ is torsion free, $G^{(n)}$ is torsion free, infinite, and it is also abelian and normal in $G$. We may then apply Lemma 3.7 to $A=G^{(n)}$, to find that there is a finite index subgroup $H$ of $G$ such that $[H, H] \cap G^{(n)}=\{1\}$. However, $H^{(n)} \subset G^{(n)}$, and it follows that $H^{(n)}$ is trivial. The induction hypothesis implies that $H$ is virtually abelian, hence so is $G$.

\section{Lamps and lighters}

\subsection{A lamplighter group in IET}

Proposition 4.1. - For all finite abelian group $A$, the group $A \backslash \mathbb{Z}$ embeds in IET.

Recall that in general, the group $A \imath G$ is the group $\left(\bigoplus_{i \in G} A\right) \rtimes G$, where $G$ acts by shifting coordinates: if $g \in G$, and $\left(a_{i}\right)_{i \in G} \in\left(\bigoplus_{i \in G} A\right)$ is an almost null sequence, $g \cdot\left(a_{i}\right)_{i \in G}=\left(a_{g^{-1} i}\right)_{i \in G}$.

We will actually describe an embedding in $\operatorname{IET}(\mathcal{D})$ for a certain domain $\mathcal{D}$. However, IET and $\operatorname{IET}(\mathcal{D})$ are isomorphic, and the choice of $\mathcal{D}$ is only for convenience.

The construction is illustrated in Figure 4.1. 


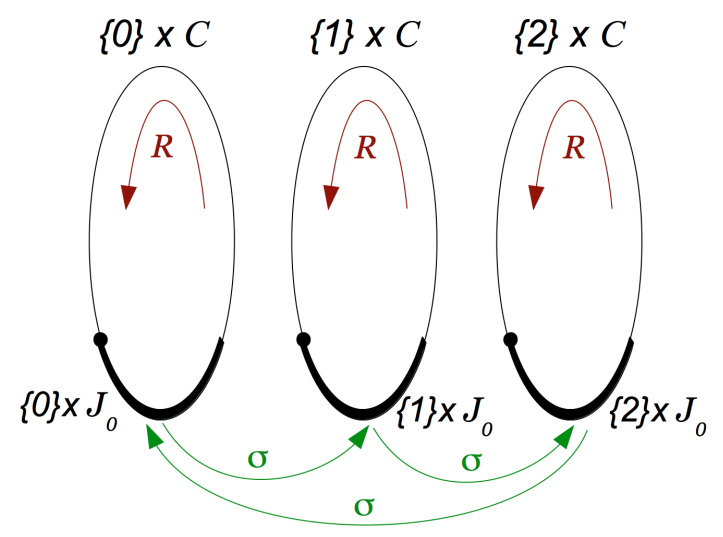

Figure 4.1. A Lamplighter group $(\mathbb{Z} / 3 \mathbb{Z}) \prec \mathbb{Z}$ in IET. The three circles $\{i\} \times \mathcal{C}$ for $i \in \mathbb{Z} / 3 \mathbb{Z}$ are visible. The transformation $R$ rotates each circle by the irrational angle $\theta$. The support of the transformation $\sigma=\sigma_{1, J_{0}}$ is the union of the bold arcs.

Proof. - Consider the domain $\mathcal{D}=A \times \mathcal{C}$ where $\mathcal{C}=\mathbb{R} / \mathbb{Z}$. Given $a \in A$ and $J \subset \mathcal{C}$ a subinterval, let $\sigma_{a, J}$ be the element of $\operatorname{IET}(\mathcal{D})$ defined for all $\left(a^{\prime}, x\right) \in A \times \mathcal{C}$ by

$$
\sigma_{a, J} \cdot\left(a^{\prime}, x\right)= \begin{cases}\left(a a^{\prime}, x\right) & \text { if } x \in J \\ \left(a^{\prime}, x\right) & \text { if } x \notin J .\end{cases}
$$

Note that the support of $\sigma_{a, J}$ is $A \times J$ when $a \neq 1$. We define $\mathcal{A}_{J}$ as the subgroup of $\operatorname{IET}(\mathcal{D})$ consisting of the elements $\sigma_{a, J}$ for $a \in A$ (note that $\mathcal{A}_{J}$ is isomorphic to $A$ as long as $J$ is non-empty).

Fix $J_{0}=\left[0,1 / 2\left[\subset \mathcal{C}\right.\right.$, and let $\mathcal{A}=\mathcal{A}_{J_{0}}$. Let $\theta \in \mathbb{R} \backslash \mathbb{Q}$, and let $R \in \operatorname{IET}(\mathcal{D})$ be the rotation by $\theta$ on each circle: $R(a, x)=(a, x+\theta)$. We claim that the group generated by $R$ and $\mathcal{A}_{J}$ is isomorphic to $A$ ? $\mathbb{Z}$.

First, one easily checks that for all $J, J^{\prime} \subset \mathcal{C}$, any element of $\mathcal{A}_{J}$ commutes with any element of $\mathcal{A}_{J^{\prime}}$ (this is because $A$ is abelian). Denote by $t$ a generator of the factor $\mathbb{Z}$ in $A$ 亿 $\mathbb{Z}$. Since $R^{k} \mathcal{A} R^{-k}=\mathcal{A}_{R^{k} J_{0}}$, there is a homomorphism $\varphi: A \imath \mathbb{Z} \rightarrow\langle R, \mathcal{A}\rangle$ sending $t$ to $R$ and sending the almost null sequence $\left(a_{i}\right)_{i \in \mathbb{Z}}$ to $\prod_{i \in \mathbb{Z}} \sigma_{a_{i}, R^{i} J_{0}}$.

To prove that $\varphi$ is injective, consider an element $g=\left(\left(a_{i}\right)_{i \in \mathbb{Z}}, t^{k}\right)$ of its kernel. Since $\varphi(g)$ sends $(a, x)$ to some $\left(a^{\prime}, x+k \theta\right)$, we get that $k=0$. This means that $\varphi(g)$ is a commuting product $\varphi(g)=\sigma_{a_{1}, J_{1}} \ldots \sigma_{a_{n}, J_{n}}$ where $J_{1}, \ldots J_{n}$ are distinct translates of $J_{0}$ by a multiple of $\theta$. We can assume 
that $n>0$ and that no $a_{i}$ is trivial. For all $x \in \mathcal{C}$, consider $\mathcal{J}_{x} \subset\{1, \ldots, n\}$ the set of indices $j \in\{1, \ldots, n\}$ such that $x \in J_{j}$. Note that when $x$ varies, $\mathcal{J}_{x}$ changes only when $x$ crosses an endpoint of some $J_{i}$, and that $\mathcal{J}_{x}$ then changes by exactly one element (this is because the $2 n$ endpoints of the $J_{i}$ 's are distinct since $\theta$ is irrational). Thus, there exist $x, x^{\prime} \in \mathcal{C}$ and $i_{0} \in\{1, \ldots n\}$ such that $\mathcal{J}_{x^{\prime}}=\mathcal{J}_{x} \cup\left\{J_{i_{0}}\right\}$. Now, for all $a \in A, \varphi(g)(a, x)=\left(a \prod_{j \in \mathcal{J}_{x}} a_{j}, x\right)$, and since $\varphi(g)$ is the identity, $\prod_{j \in \mathcal{J}_{x}} a_{j}=1$. Similarly, $\prod_{j \in \mathcal{J}_{x^{\prime}}} a_{j}=1$, so $a_{i_{0}}=1$, a contradiction.

The argument above immediately generalizes to $A \prec \mathbb{Z}^{d}$, by replacing the rotation of angle $\theta$ by $d$ rotations of rationally independant angles. We thus get:

Proposition 4.2. - For all $d \geqslant 1$, and all finite abelian group $A, A \gtrless \mathbb{Z}^{d}$ embeds in IET.

Remark 4.3. - Given $G<$ IET, and $A$ a finite abelian group, we don't know when the group $A$ $G$ embeds in IET.

\subsection{Lamps must commute}

We now put restrictions on which wreath products may embed in IET. Note that if a group $A$ contains an infinite order element, then $A$ ? $\mathbb{Z}$ contains the torsion-free solvable group $\mathbb{Z} \imath \mathbb{Z}$ which does not embed in IET by Theorem 3.1.

THEOREM 4.4. - Let $L=F \nmid \mathbb{Z}$ with $F$ finite non-abelian. Then $L$ does not embed in IET.

We will use several times the observation (see [3, Lem. 6.2]) that for any finitely generated subgroup $G$ of $\operatorname{IET}(\mathcal{D})$, the orbit of any point of the domain $\mathcal{D}$ has polynomial growth in the following sense: given a finite generating set and the corresponding word metric on $G$, denoting by $B_{R}$ the ball of radius $R$ in $G$, there exists a polynomial $P$ such that for all $x \in \mathcal{D}$ and all $R \geqslant 0$, $\#\left(B_{R} \cdot x\right) \leqslant P(R)$.

The theorem will be proved by showing that if $L$ did embed in IET, there would exist an orbit with exponential growth.

If $E \subset[0,1)$ has positive measure and if $T$ is any element of IET, then by Poincaré recurrence Theorem, the orbit of almost every $x \in E$ comes back to $E$. We will need a more precise estimate, that comes from Birkhoff theorem. 
Lemma 4.5. - Let $E \subset[0,1)$ have positive measure, and let $T$ be any element in IET.

Then there exists $x_{0} \in[0,1)$, and constants $\alpha>0, \beta>0$, such that for all $n$,

$$
\#\left\{i \mid 0 \leqslant i<n, T^{i}(x) \in E\right\} \geqslant \alpha n-\beta .
$$

Proof. - Assume first that the Lebesgue measure $\mu$ is ergodic, and apply Birkhoff theorem to the characteristic function $f$ of $E$. Then for almost every $x, \frac{1}{n} \sum_{i=0}^{n-1} f\left(T^{i}(x)\right) \rightarrow \mu(E)$, so for $n$ large enough, $\frac{1}{n} \sum_{i=0}^{n-1} f\left(T^{i}(x)\right) \geqslant$ $\frac{1}{2} \mu(E)$, so $\#\left\{i \mid 0 \leqslant i \leqslant n, T^{i}(x) \in E\right\} \geqslant \frac{1}{2} \mu(E) n$ for $n$ large enough, and the result holds.

If the Lebesgue measure is not ergodic, Birkhoff theorem still says that the limit exists almost everywhere, and the limit is a $T$-invariant function $l(x)$ having the same average as $f$, that is $\int l(x) d x=\mu(E)$ (see [8] for instance). It follows that there exist points where $l(x) \geqslant \mu(E) / 2$, and the proof works the same.

We prove an abstract algebraic lemma that relates the stabilizer of a point in a product of groups to coordinate-wise stabilizers.

Lemma 4.6. - Consider some groups $F_{i}$, and an action of $F=F_{1} \times \cdots \times$ $F_{n}$ on a set $X$. Let $x \in X, \operatorname{Stab}(x)$ its stabilizer in $F$, and $S_{i}=F_{i} \cap \operatorname{Stab}(x)$. Let $N_{i}$ the normalizer of $S_{i}$ in $F_{i}$.

Then $\operatorname{Stab}(x) \subset N_{1} \times \cdots \times N_{n}$.

In particular, if no $S_{i}$ is normal in $F_{i}$, then \#F. $x \geqslant 2^{n}$.

Proof. - Note that $N_{1} \times \cdots \times N_{n}=\cap_{i} N_{F}\left(S_{i}\right)$, so we have to prove that for each $i$, any $g \in \operatorname{Stab}(x)$ normalizes $S_{i}$. If $g \in \operatorname{Stab}(x)$, then $g$ normalizes both $\operatorname{Stab}(x)$ and $F_{i}$ (because $F_{i} \triangleleft F$ ), hence normalizes their intersection, namely $S_{i}$.

Let us prove the last comment. The given assumption says that for all $i$, $\left[F_{i}: N_{i}\right] \geqslant 2$ so \#F.x $=[F: \operatorname{Stab}(x)] \geqslant 2^{n}$.

To prove the theorem, consider $L=F_{0} \prec \mathbb{Z}$ for some finite non-abelian group $F_{0}$. We assume that $L$ embeds in IET and argue towards a contradiction. Let $t$ be a generator of $\mathbb{Z}$ viewed as a subgroup of $L$, and write $F_{i}=F_{0}^{t^{i}}$. We equip the group $G$ with the word metric corresponding to the generating set $\{t\} \cup F_{0}$. The subgroup $F_{0} \times F_{1} \times \cdots \times F_{n-1}$ is contained in the ball of radius $3 n$. We identify $L$ with the corresponding subgroup of IET. Since orbits grow polynomially [3, Lem. 6.2$]$, for any $x \in[0,1)$, the orbit of $x$ under $F_{0} \times \cdots \times F_{n-1}$ has to be bounded by a polynomial in $n$.

Proposition 4.7. - For all $x \in[0,1), \operatorname{Stab}_{F_{0}}(x) \triangleleft F_{0}$. 
Proof. - Otherwise, let $E \subset[0,1)$ be the set of points where $\operatorname{Stab}_{F_{0}}(x) \Varangle$ $F_{0}$. Since $F_{0}$ is a finite group, this is a subdomain of $[0,1)$, and it has positive measure. We apply Birkhoff theorem (in the form of Lemma 4.5), and get that there exists $x \in[0,1), \alpha, \beta>0$, such that, for all $n$, there exists $k_{n} \geqslant$ $\alpha n-\beta$, and some indices $0 \leqslant i_{1}<\cdots<i_{k_{n}} \leqslant n$ such that $t^{i_{j}}(x) \in E$. Applying the algebraic Lemma 4.6 to $F=F_{i_{1}} \times \cdots \times F_{i_{k_{n}}}$ we get that $\#(F . x) \geqslant 2^{k_{n}} \geqslant 2^{\alpha n-\beta}$. Since $F$ is contained in a ball of linear radius in $L$, this contradicts polynomial growth of orbits.

We now prove another algebraic lemma.

Lemma 4.8. - Consider an action of $F=F_{1} \times \cdots \times F_{n}$ on a set $X$. Let $x \in X, \operatorname{Stab}(x)$ its stabilizer in $x$, and $S_{i}=\operatorname{Stab}(x) \cap F_{i}$ its stabilizer in $F_{i}$.

Assume that $\operatorname{Stab}(x) \triangleleft F$ (in particular $\left.S_{i} \triangleleft F_{i}\right)$. Consider $Z\left(F_{i} / S_{i}\right)$ the center of the quotient group $F_{i} / S_{i}$, and $Q_{i}=\left(F_{i} / S_{i}\right) / Z\left(F_{i} / S_{i}\right)$.

Then the natural epimorphism $F_{1} \times \cdots \times F_{n} \rightarrow Q_{1} \times \cdots \times Q_{n}$ factors through an epimorphism $F / \operatorname{Stab}(x) \rightarrow Q_{1} \times \cdots \times Q_{n}$.

In particular, if all groups $F_{i} / S_{i}$ are non-abelian, each $Q_{i}$ is non-trivial, and $\# F . x=\# F / \operatorname{Stab}(x) \geqslant 2^{n}$.

Proof. - Consider $g=\left(g_{1}, \ldots, g_{n}\right) \in \operatorname{Stab}(x)$, and denote by $\bar{g}_{i}$ the image of $g_{i} \in F_{i} / S_{i}$. We have to prove that $\bar{g}_{i}$ is central in $F_{i} / S_{i}$, in other words that for all $a \in F_{i},\left[g_{i}, a\right] \in S_{i}$. Since $S_{i}=\operatorname{Stab}(x) \cap F_{i}$ is normal in $F,\left[g_{i}, a\right]=g_{i}\left(a g_{i}^{-1} a^{-1}\right) \in S_{i}$. The lemma follows.

Proof of Theorem 4.4. - Fix $k \geqslant 1$ and consider the subgroup $L^{\prime} \subset L$ generated by $t^{k}$, and $F_{0} \times \cdots \times F_{k-1}$. Clearly, $L^{\prime} \simeq F_{0}^{\prime} \prec \mathbb{Z}$ where $F_{0}^{\prime}=$ $F_{0} \times \cdots \times F_{k-1}$. Applying Proposition 4.7 to $L^{\prime}$, we get that for all $x$, and all $k$, the stabilizer of $x$ in $F_{0} \times \cdots \times F_{k-1}$ is normal in $F_{0} \times \cdots \times F_{k-1}$.

Let $E \subset[0,1)$ be the set of points $x$ where $F_{0} / \operatorname{Stab}(x)$ is non-abelian. If $E$ is empty, any commutator of $F_{0}$ acts trivially in $[0,1)$, so $F_{0}$ does not act faithfully, a contradiction. So $E$ is a non-empty subdomain and has positive measure. By Lemma 4.5, there exists $x \in[0,1), \alpha, \beta>0$, such that, for all $n$, there exists $k_{n} \geqslant \alpha n-\beta$, and some indices $0 \leqslant i_{1}<\cdots<i_{k_{n}} \leqslant n$ such that $t^{i_{j}}(x) \in E$.

Applying the second algebraic Lemma 4.8 to $F=F_{i_{1}} \times \cdots \times F_{i_{k_{n}}}$ we get that $\# F . x \geqslant 2^{\alpha n-\beta}$. This contradicts polynomial growth of orbits as above. 


\subsection{Uncountably many solvable groups, via lamplighter-lighters}

We finish by an abundance result among solvable subgroups of IET (necessarily with a lot of torsion in view of Theorem 3.1).

THEOREM 4.9. - There exist uncountably many isomorphism classes of 3-generated solvable subgroups of derived length 3 in IET.

These will be obtained as iterated lamplighter-like constructions. Again we will be free to choose the domain $\mathcal{D}$ for convenience.

For the time being, take $\mathcal{D}$ an arbitrary domain. Let $G<\operatorname{IET}(\mathcal{D})$ and $(A,+)$ a finite abelian group. The following construction generalizes the construction showing that lamplighter groups embed. In this case, $G$ would be the cyclic group generated by an irrational rotation on a circle $\mathcal{D}$.

Let $\mathcal{F}=A^{\mathcal{D}}$ be the additive group of all functions on $\mathcal{D}$ with values in $A$. Given $a \in A$ and $J \subset \mathcal{D}$, we denote by $a \mathbb{1}_{J} \in \mathcal{F}$ the function defined by $a \mathbb{1}_{J}(x)=a$ if $x \in J$, and $a \mathbb{1}_{J}(x)=0$ otherwise. The group $G$ acts on $\mathcal{F}$ by precomposition. Given a subdomain $J \subset \mathcal{D}$, let $\mathcal{F}_{J} \subset \mathcal{F}$ be the smallest $G$-invariant additive subgroup containing the functions $a \mathbb{1}_{J}$ for $a \in A$.

Proposition 4.10 (Lamplighter-like construction). - Let $G$ be a subgroup of $\operatorname{IET}(\mathcal{D}), A$ a finite abelian group, and $J \subset \mathcal{D}$. Then $\mathcal{F}_{J} \rtimes G$ embeds in IET.

Remark 4.11. - In the particular case of Proposition 4.1, we proved that $\mathcal{F}_{J}$ is isomorphic to $\bigoplus_{g \in G} A$. In general, there is a natural morphism from $\bigoplus_{g \in G} A$ to $\mathcal{F}_{J}$, but it might be non-injective. One easily constructs examples using a group $G$ that does not act freely on $\mathcal{D}$.

Proof. - Take $\mathcal{D}^{\prime}=A \times \mathcal{D}$. Embed $G$ in $\operatorname{IET}\left(\mathcal{D}^{\prime}\right)$ by setting for all $g \in G$, $g(a, x)=(a, g(x))$.

For all $b \in A$, consider $\sigma_{b} \in \operatorname{IET}\left(\mathcal{D}^{\prime}\right)$ defined by $\sigma_{b}(a, x)=(b+a, x)$ if $x \in J$, and by $\sigma_{b}(a, x)=(a, x)$ if $x \notin J$.

We claim that the subgroup $G^{\prime}$ generated by $G$ and $\left\{\sigma_{b} \mid b \in A\right\}$ is isomorphic to $\mathcal{F}_{J} \rtimes G$.

Consider the subset $H \subset \operatorname{IET}\left(\mathcal{D}^{\prime}\right)$ of all $T \in I E T\left(\mathcal{D}^{\prime}\right)$ such that there exists some transformation $g \in G$ and some $f \in \mathcal{F}_{J}$ such that $T(a, x)=$ $(a+f(x), g(x))$. It is clear that $H$ is a subgroup of $\operatorname{IET}\left(\mathcal{D}^{\prime}\right)$, that $H$ contains $G^{\prime}$, and that $G^{\prime}=H$. Finally, the fact that $H \simeq \mathcal{F}_{J} \rtimes G$ is also clear.

Let $\mathcal{D}_{0}=\mathbb{R} / \mathbb{Z}$, and $I=\left[0,1 / 2\left[\subset \mathcal{D}_{0}\right.\right.$. Fix once and for all $\alpha \notin \mathbb{Q}, \rho_{\alpha}$ the rotation of $\mathcal{D}_{0}$ of angle $\alpha$. Let $G$ be the lamplighter group $(\mathbb{Z} / 3 \mathbb{Z}) \prec \mathbb{Z}$ realised 


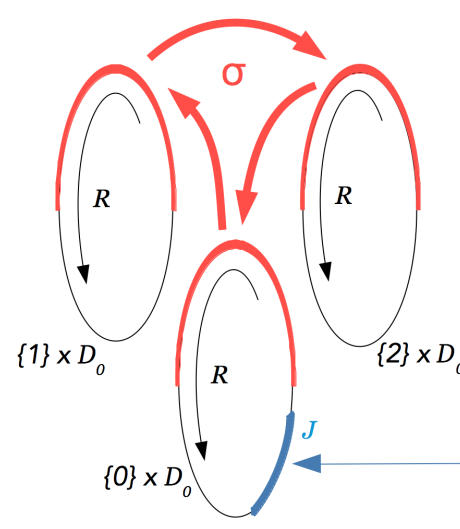

$\{0\} \times D$

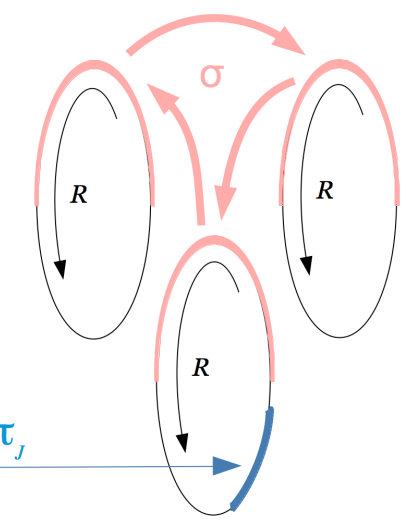

$\{b\} \times D$

Figure 4.2. A Lamplighter-like construction in IET producing the examples of Theorem 4.9: on the left, the domain $\mathcal{D}=\mathbb{Z} / 3 \mathbb{Z} \times \mathcal{D}_{0}$, and the Lamplighter group $G=\mathbb{Z} / 3 \mathbb{Z} 2 \mathbb{Z}$ generated by a cyclic swap on the three intervals $\mathbb{Z} / 3 \mathbb{Z} \times I$ (in bold red) and by the simultaneous rotation $R$. On the right, $\{b\} \times \mathcal{D}$ is the duplicated copy of $\mathcal{D}$, with the swap $\tau_{J}$ on $J$ and its copy. The group $G$ acts diagonally on $\mathcal{D}^{\prime}=\mathbb{Z} / 2 \mathbb{Z} \times \mathcal{D}$. The group $\left(\bigoplus_{G} \mathbb{Z} / 2 \mathbb{Z}\right) \rtimes G$ acts on $\mathcal{D}^{\prime}$ but its image $H_{J}$ in $\operatorname{IET}\left(\mathcal{D}^{\prime}\right)$ depends on $J$.

as a subgroup of $\operatorname{IET}(\mathcal{D})$ for $\mathcal{D}=(\mathbb{Z} / 3 \mathbb{Z}) \times \mathcal{D}_{0}$ as in Proposition 4.1. We denote by $R$ the rotation $(a, x) \mapsto\left(a, \rho_{\alpha}(x)\right)$ on $\mathcal{D}$.

We now perform this lamplighter-like construction a second time, starting with $G$ acting on $\mathcal{D}$, with $A=\mathbb{Z} / 2 \mathbb{Z}$ and with some $J \subset\{0\} \times \mathcal{D}_{0} \subset \mathcal{D}$. We recall that $\mathcal{F}_{J}$ is the subgroup of the group of functions from $\mathcal{D}$ to $A=\mathbb{Z} / 2 \mathbb{Z}$ as above. Define $H_{J}=\mathcal{F}_{J} \rtimes G$ the lamplighter-like group thus obtained, on $\mathcal{D}^{\prime}=(\mathbb{Z} / 2 \mathbb{Z}) \times \mathcal{D}$. See Figure 4.2 .

Proposition 4.12. - Assume that $J_{1}, J_{2} \subset\{0\} \times \mathcal{D}_{0} \subset \mathcal{D}$, and that $\left|J_{1}\right|,\left|J_{2}\right|<\frac{1}{2}$.

If $H_{J_{1}} \simeq H_{J_{2}}$ then $\left|J_{1}\right| \in \operatorname{Vect}_{\mathbb{Q}}\left(1, \alpha, a_{2}, b_{2}\right)$, where $a_{2}, b_{2} \in \mathbb{R} / \mathbb{Z}$ are the endpoints of $J_{2}$.

Denote by $\mathcal{E}_{I}$ and $\mathcal{E}_{J_{i}} \subset \mathbb{R} / \mathbb{Z}$ the orbit under the rotation $\rho_{\alpha}$ of the endpoints of $I$ and $J_{i}$ respectively (i.e. $\mathcal{E}_{I}=\left\{0, \frac{1}{2}\right\}+\alpha \mathbb{Z}, \mathcal{E}_{J_{i}}=\left\{a_{i}, b_{i}\right\}+\alpha \mathbb{Z}$ ). 
Lemma 4.13. - If $H_{J_{1}} \simeq H_{J_{2}}$, then there exist two subdomains $K, K^{\prime} \subset$ $\mathbb{R} / \mathbb{Z}$ with endpoints in $\mathcal{E}_{J_{2}}$ and $\mathcal{E}_{I}$ respectively and $\varepsilon \in\{ \pm 1\}$ such that

$$
\forall n \in \mathbb{Z}, \quad \rho_{\alpha}^{n}(I) \cap J_{1} \neq \emptyset \Longleftrightarrow \rho_{\alpha}^{\varepsilon n}(K) \cap K^{\prime} \neq \emptyset .
$$

Proof of Proposition 4.12 from Lemma 4.13. - We have that $\rho_{\alpha}^{n}(I) \cap$ $J_{1} \neq \emptyset$ if and only if $n \alpha \in J_{1}-I$, and that $\rho_{\alpha}^{\varepsilon n}(K) \cap K^{\prime} \neq \emptyset$ if and only if $n \alpha \in \varepsilon\left(K^{\prime}-K\right)$.

By Birkhoff theorem, we get that $\left|J_{1}-I\right|=\left|K^{\prime}-K\right|$. Since $\left|J_{1}\right|<\frac{1}{2}=|I|$, $\left|J_{1}-I\right|=\frac{1}{2}+\left|J_{1}\right|$.

On the other hand, since $K^{\prime}-K$ is a union of intervals whose endpoints are in $\mathcal{E}_{I}-\mathcal{E}_{J_{2}} \subset \operatorname{Vect}_{\mathbb{Q}}\left(1, \alpha, a_{2}, b_{2}\right)$, the proposition follows.

We view $H_{J}$ as the semidirect product $\mathcal{F}_{J} \rtimes G$. Thus each element $h \in H_{J}$ can be written (uniquely) under the form $h=g \tau$ with $\tau \in \mathcal{F}_{J}, g \in G$. Now we view $G$ as a group of interval exchange transformations on $\mathcal{D}=$ $(\mathbb{Z} / 3 \mathbb{Z}) \times(\mathbb{R} / \mathbb{Z})$. It is generated by the rotation $R:(a, x) \mapsto(a, x+\alpha)$, and by the lamp element $\sigma \in \mathcal{S}$ (of order 3 ) that sends $(a, x) \in(\mathbb{Z} / 3 \mathbb{Z}) \times(\mathbb{R} / \mathbb{Z})$ to $(a+1, x)$ for $x \in I$, and is the identity otherwise (see left part of figure 4.2). Since $G$ is a lamplighter group, any $g \in G$ can be written uniquely as $R^{n} S_{f}$ where $S_{f}$ is an IET of the form $(a, x) \rightarrow(f(x)+a, x)$ for some function $f: \mathbb{R} / \mathbb{Z} \rightarrow \mathbb{Z} / 3 \mathbb{Z}$. We denote by $\mathcal{S}=\left\{S_{f}\right\}$ the 3 -torsion abelian group of lamps of $G$. It is freely generated by the $\langle R\rangle$-conjugates of $\sigma$. Thus any $h \in H_{J}$ is written in a unique way as $h=R^{n} S_{f} \tau$ as above.

The kernel of the natural map $H_{J} \rightarrow \mathbb{Z}$ is the torsion group $N=\mathcal{F}_{J} \mathcal{S}$. It is exactly the set of elements of finite order.

Denote by $b$ be the generator of $\mathbb{Z} / 2 \mathbb{Z}$. Given $K \subset \mathcal{D}$ denote by $\tau_{K}=$ $b \mathbb{1}_{K} \in \mathcal{F}$. For certain $K$ (for instance $K=J$ ), $\tau_{K} \in \mathcal{F}_{J}$ and thus is in $H_{J}$.

FACT 4.14. - If $K \subset\{0\} \times \mathcal{D}_{0}$, then $\left[\sigma, \tau_{K}\right]=1$ if and only if $K \cap I=\emptyset$.

Proof. - From the definition of the semidirect product, $\sigma \tau_{K} \sigma^{-1}=$ $b \mathbb{1}_{\sigma(K)}$. Hence $\left[\sigma, \tau_{K}\right]=1$ if and only if $\sigma(K)=K$. If $K \cap I \neq \emptyset$, then $\sigma(K)$ contains a point outside $\{0\} \times \mathcal{D}_{0}$, so $\sigma(K) \neq K$.

FACT 4.15. - For any $\tau, \tau^{\prime} \in \mathcal{F}_{J}$ and any $h \in H_{J},\left[h \tau, \tau^{\prime}\right]=\left[h, \tau^{\prime}\right]$.

Proof. - This is is an immediate consequence of the commutation of $\tau$ with $\tau^{\prime}$.

Proof of Lemma 4.13. - Let $\varphi: H_{J_{1}} \rightarrow H_{J_{2}}$ be an isomorphism. We first note that $\mathcal{F}_{J} \triangleleft H_{J}$ is precisely the set of elements $g \in H_{J}$ such that $g^{2}=1$. Moreover, the set of elements of finite order in $H_{J}$ is the subgroup $N=\mathcal{F}_{J} \mathcal{S}$ (but there are exotic elements of order 3 ). 
Thus $\varphi\left(\tau_{J_{1}}\right) \in \mathcal{F}_{J_{2}}$ can be viewed as a function $\tau: \mathcal{D} \rightarrow \mathbb{Z} / 2 \mathbb{Z}$, and $\varphi(\sigma)$ as an element $S^{\prime} \tau^{\prime}$ for some $S^{\prime} \in \mathcal{S}$, and $\tau^{\prime} \in \mathcal{F}_{J}$. Now $\varphi(R)$ generates $G_{J_{2}}$ modulo the torsion subgroup, so $\mathcal{F}_{J_{2}}$, so $\varphi(R)=R^{\varepsilon} S^{\prime \prime} \tau^{\prime \prime}$ for some function $\tau^{\prime \prime} \in \mathcal{F}_{J_{2}}, S^{\prime \prime} \in \mathcal{S}$, and $\varepsilon= \pm 1$.

In what follows, we use the notation $g^{h}$ for $h g h^{-1}$. Fix $n \in \mathbb{Z}$ and consider the commutator $C=\left[\sigma, \tau_{J_{1}}^{R^{n}}\right]=\left[\sigma, \tau_{R^{n}\left(J_{1}\right)}\right]$. By Fact 4.14, $C$ is trivial if and only if $R^{n}\left(J_{1}\right) \cap I=\emptyset$. On the other hand, by Fact 4.15 ,

$$
\varphi(C)=\left[S^{\prime} \tau^{\prime}, \tau^{\left(R^{\varepsilon} S^{\prime \prime} \tau^{\prime \prime}\right)^{n}}\right]=\left[S^{\prime}, \tau^{\left(R^{\varepsilon} S^{\prime \prime} \tau^{\prime \prime}\right)^{n}}\right] .
$$

There exists $\tau^{\prime \prime \prime} \in \mathcal{F}_{J_{2}}$ such that $\left(R^{\varepsilon} S^{\prime \prime} \tau^{\prime \prime}\right)^{n}=\left(R^{\varepsilon} S^{\prime \prime}\right)^{n} \tau^{\prime \prime \prime}$, and since $\mathcal{F}_{J_{2}}$ is abelian, $\tau^{\left(R^{\varepsilon} S^{\prime \prime} \tau^{\prime \prime}\right)^{n}}=\tau^{\left(R^{\varepsilon} S^{\prime \prime}\right)^{n}}$. Hence, $\varphi(C)=1$ if and only if $\left[S^{\prime\left(R^{\varepsilon} S^{\prime \prime}\right)^{-n}}, \tau\right]=1$. A similar calculation in $G$ shows that $S^{\prime\left(R^{\varepsilon} S^{\prime \prime}\right)^{-n}}=$ $S^{R^{-\varepsilon n} S^{\prime \prime \prime}}=S^{R^{-\varepsilon n}}$, so $\varphi(C)=1$ if and only if $\left[S^{\prime}, \tau^{R^{\varepsilon n}}\right]=1$.

Given $x \in \mathbb{R} / \mathbb{Z}$, we call the fiber of $x$ the 3 point set $F_{x}=\{(a, x) \mid a \in$ $\mathbb{Z} / 3 \mathbb{Z}\} \subset \mathcal{D}$. Let $K \subset \mathbb{R} / \mathbb{Z}$ be the set of points $x$ such that $\tau$ is non constant on the fiber of $x$. Since $\tau \in \mathcal{F}_{J_{2}}, K$ is a union of intervals with endpoints in $\mathcal{E}_{J_{2}}$. Write the transformation $S^{\prime}$ as $(a, x) \mapsto(a+f(x), x)$ for some function $f: \mathbb{R} / \mathbb{Z} \rightarrow \mathbb{Z} / 3 \mathbb{Z}$, and let $K^{\prime}$ be the support of $f$. It is a union of intervals with endpoints in $\mathcal{E}_{I}$.

We claim that $\left[S^{\prime}, \tau^{R^{\varepsilon n}}\right]=1$ if and only if $R^{\varepsilon n}(K) \cap K^{\prime}=\emptyset$. Note that $S^{\prime}$ preserves each fiber. We view $\tau$ as an element in the additive group $\mathcal{F}$ of functions on $\mathcal{D}$ with values in $\mathbb{Z} / 2 \mathbb{Z}$, and $S^{\prime}, R$ as transformations of $\mathcal{D}$, so that $\left[S^{\prime}, \tau^{R^{\varepsilon n}}\right]=1$ if and only if $\tau \circ R^{\varepsilon n} \circ S^{\prime}=\tau \circ R^{\varepsilon n}$. So fix $x \in \mathbb{R} / \mathbb{Z}$, and $F_{x}$ its fiber. If $x \notin K^{\prime}$, then $S^{\prime}$ acts as the identity on $F_{x}$, so $\tau \circ R^{\varepsilon n} \circ S^{\prime}=\tau \circ R^{\varepsilon n}$ in retriction to $F_{x}$. If $x \notin R^{\varepsilon n}(K)$, then $\tau^{R^{\varepsilon n}}$ is a constant function on $F_{x}$, and the same conclusion holds. If on the contrary $x \in R^{\varepsilon n}(K) \cap K$, then $\tau^{R^{\varepsilon n}}$ is not constant on $F_{x}$ and since $S^{\prime}$ acts transitively on $F_{x}, \tau^{R^{\varepsilon n}} \circ S^{\prime}$ does not coincide with $\tau^{R^{\varepsilon n}}$ on $F_{x}$. This proves the claim and concludes the proof.

We can finally prove Theorem 4.9.

Proof of Theorem 4.9. - Let $J_{x}=\left[0, x\left[\right.\right.$ for $x<\frac{1}{2}$. Proposition 4.12 shows that given $x$, there are at most countably many $y<\frac{1}{2}$ such that $H_{J_{x}} \simeq H_{J_{y}}$. This gives uncountably many isomorphism classes of groups.

Since $G$ is metabelian, $H_{J_{x}}$ is solvable of derived length at most 3. Since there are only countably many isomorphism classes of finitely generated metabelian groups, $H_{J_{x}}$ has derived length exactly 3 for uncountably many values of $x$. 
François Dahmani, Koji Fujiwara and Vincent Guirardel

\section{Acknowledgement}

We would like to thank the anonymous referee for suggestions that improved the exposition. The first and third authors acknowledge support from ANR-11-BS01-013 while this work was started, and are supported by the Institut Universitaire de France. The second named author is supported in part by Grant-in-Aid for Scientific Research (No. 15H05739). The author thanks the Centre Henri Lebesgue ANR-11-LABX-0020-01 for creating an attractive mathematical environment. This material is based upon work supported by the National Science Fondation under grant No. DMS-1440140 while the authors were in residence at the Mathematical Science Research Institute in Berkeley California, during the Fall 2016 semester.

\section{Bibliography}

[1] Y. DE Cornulier, "Groupes pleins-topologiques (d'après Matui, Juschenko, Monod, ...)", in Séminaire Bourbaki. Volume 2012/2013, Astérisque, vol. 361, Société Mathématique de France, 2014, Exp. No. 1064, p. 183-223.

[2] - "Commensurating actions for groups of piecewise continuous transformations", https://arxiv.org/abs/1803.08572, 2018.

[3] F. Dahmani, K. Fujiwara \& V. Guirardel, "Free groups of interval exchange transformations are rare", Groups Geom. Dyn. 7 (2013), no. 4, p. 883-910.

[4] D. Gaboriau, G. Levitt \& F. Paulin, "Pseudogroups of isometries of $\mathbb{R}$ and Rips' theorem on free actions on $\mathbb{R}$-trees", Isr. J. Math. 87 (1994), no. 1-3, p. 403-428.

[5] H. Imanishi, "On codimension one foliations defined by closed one-forms with singularities", J. Math. Kyoto Univ. 19 (1979), no. 2, p. 285-291.

[6] K. Juschenko, N. Matte Bon, N. Monod \& M. de la Salle, "Extensive amenability and an application to interval exchanges", Ergodic Theory Dyn. Syst. 38 (2018), no. 1, p. 195-219.

[7] K. Juschenko \& N. Monod, "Cantor systems, piecewise translations and simple amenable groups", Ann. Math. 178 (2013), no. 2, p. 775-787.

[8] Y. Katznelson \& B. Weiss, "A simple proof of some ergodic theorems", Isr. J. Math. 42 (1982), no. 4, p. 291-296.

[9] M. Krasner \& L. Kaloujnine, "Produit complet des groupes de permutations et le problème d'extension de groupes III", Acta Sci. Math. 14 (1951), p. 69-82.

[10] C. F. Novak, "Discontinuity-growth of interval-exchange maps", J. Mod. Dyn. 3 (2009), no. 3, p. 379-405. 